\title{
Training in Endoscopy: Endoscopic Ultrasound
}

\author{
Chang Min Cho \\ Department of Internal Medicine, Kyungpook National University Medical Center, Kyungpook National University College of Medicine, Daegu, \\ Korea
}

Endoscopic ultrasound (EUS) has been recently established as an indispensable modality for the diagnosis and management of pancreatobiliary and gastrointestinal (GI) disorders. EUS proficiency requires both cognitive and technical abilities, including an understanding of the appropriate indications, the performance of appropriate evaluations before and after the procedure, and the management of procedure-related complications. An increasing demand for skills to handle a growing range of interventional EUS procedures and a continual shortage of EUS training programs are two major obstacles for EUS training. Acquiring the skills necessary to comprehend and conduct EUS often requires training beyond the scope of a standard GI fellowship program. In addition to traditional formal EUS training and preceptorships, regular short-term intensive EUS training programs that provide training at various levels may help EUS practitioners improve and maintain EUS-related knowledges and skills. Theoretical knowledge can be acquired from lectures, textbooks, atlases, slides, videotapes, digital video discs, interactive compact discs, and websites. Informal EUS training is generally based on 1- or 2-day intensive seminars, including didactic lectures, skills demonstrated by expert practitioners through live video-streaming of procedures, and hands-on learning using animal or phantom models. Clin Endosc 2017;50:340-344

Key Words: Endosonography; Training support; Competency; Hands-on

\section{INTRODUCTION}

Since first introduction into clinical practice in the early 1980s, endoscopic ultrasound (EUS) has been established as an indispensable diagnostic and therapeutic modality for pancreatobiliary and gastrointestinal (GI) disorders. ${ }^{1,2}$ However, it is considered one of the most technically challenging and complicated endoscopic procedures for the experienced endoscopist to perform and for the trainee to learn, because both cognitive and technical abilities are required for endoscopic manipulation and ultrasonographic interpretation. Over the past few decades, remarkable innovations in EUS

Received: May 4, 2017 Revised: July 20, 2017

Accepted: July 21, 2017

Correspondence: Chang Min Cho

Department of Internal Medicine, Kyungpook National University Medical Center, Kyungpook National University College of Medicine, 807 Hokuk-ro, Buk-gu, Daegu 41404, Korea

Tel: +82-53-200-2608, Fax: +82-53-200-2028, E-mail: cmcho@knu.ac.kr

(cc) This is an Open Access article distributed under the terms of the Creative Commons Attribution Non-Commercial License (http://creativecommons.org/ licenses/by-nc/3.0) which permits unrestricted non-commercial use, distribution, and reproduction in any medium, provided the original work is properly cited. technology, more versatile EUS equipment, and echoendoscopes with larger operating channel have resulted in the deployment of various interventional accessories. This has led to considerable progress in the utility of EUS, including its use in complex interventional procedures; for example, pancreatobiliary drainage, creation of anastomosis, vascular access, and tumor ablation. Since EUS applications have become increasingly recognized by other clinical practitioners, the demand for well-trained endosonographers has increased. Although EUS has substantially evolved in technology, research, and performance, unlike esophagogastroduodenoscopy and colonoscopy, the practice of EUS is still far from being part of every endoscopy suite due to its high set-up cost and relative scarcity of credentialed EUS experts. ${ }^{3}$ EUS training faces two major obstacles to be overcome: an increasing demand for skills to handle a growing range of interventional EUS procedures and a continual shortage of EUS training programs. ${ }^{4}$

This section reviews the progress and status of EUS training programs in Western and Asian regions, as well as other available informal training programs and modalities for EUS training. 


\section{PROGRESS OF EUS TRAINING PROGRAMS IN WESTERN COUNTRIES}

As an advanced endoscopic procedure, EUS procedures require substantial technical skill and extensive knowledge of intraabdominal anatomy. Significant time and effort are also required to learn EUS procedures. Acquisition of the skills necessary to understand and conduct EUS often requires training beyond the scope of a standard GI fellowship program. Although a formal fellowship in an advanced endoscopy center has always been perceived as the best way to help beginners acquire knowledge and skills for EUS procedures, such opportunities remain limited for most trainees and there is no consensus about the established EUS training environment or the adequacy of available training resources. The American Society for Gastrointestinal Endoscopy (ASGE) has published guidelines for EUS training and subsequent revisions since $1999 .^{5}$ The ASGE recommends that EUS training should be reserved for fellows who have achieved competence in routine endoscopic procedures during the training course of a GI fellowship and completed at least 24 months of a standard GI fellowship (or equivalent training). However, few academic centers in the US are available and they sometimes provide dual or separate training courses for endoscopic retrograde cholangiopancreatography (ERCP) and EUS during an additional fourth year of training for advanced procedures. In the early 2000s, EUS training was available in 28 centers in the US, one in Canada, and some European academic centers in Spain and Italy, producing a total of 30-35 expert endosonographers per year.

The training course is dependent on case volume during training and duration of experience. ${ }^{8}$ In certain unusual circumstances, a trainee may acquire the necessary skills for EUS in a short fellowship training, given adequate case volume and the necessary knowledge and skills for advanced endoscopy. To date, there have been few published reports on the number of procedures required to achieve EUS competence. The minimum number of procedures required to achieve competency is dependent on a trainee's skills and knowledge of ultrasound principles, as well as the overall quality of the training experience. To establish a certain level of EUS competence, a general consensus from expert endosonographers suggested that luminal EUS (T-staging and subepithelial lesions) requires at least 3-6 months of training, whereas pancreatobiliary EUS and fine-needle aspiration (FNA) may require up to 1 year of intensive training. ${ }^{9-11}$ ASGE has recommended 190 supervised EUS procedures (including 75 pancreatobiliary, 75 mucosal cancer staging, and 40 subepithelial cases) and 50 EUS-FNA procedures (including 25 pancreatic cases) for training and granting privileges of EUS. ${ }^{11}$ Whereas, the working group mandated by the British Society of Gastroenterology recommended that trainees should complete 250 EUS procedures, including 80 luminal cancers, 20 subepithelial lesions, and 150 pancreatobiliary cases (at least half of which are pancreatic lesions). ${ }^{12}$ The Forum on Canadian Endoscopic Ultrasound, a national Canadian meeting held annually since 2013, recently proposed guidelines stating that trainees undergo hands-on training in at least 250 supervised cases including at least 50 FNA, 100 pancreatic cases, 25 rectal cases, and at least 10 celiac plexus blocks/neurolysis procedures. ${ }^{13}$

However, the adequacy of EUS training and number of procedures in 3-year and advanced GI fellowships remain uncertain. In a survey of fellowship programs for EUS training from US GI fellowship program directors, ${ }^{14}$ fellowship programs were assessed to meet ASGE guidelines according to the presence or absence, type, and extent of EUS training. The data were analyzed based on the annual total EUS volume for 3-year and/or advanced-year fellows. Among 3-year fellows, $55 \%$ received $\leq 3$ months' training, while $43 \%$ received no actual hands-on EUS experience, and $61 \%$ did not learn EUS-guided FNA. Additionally, 20\% of advanced fellows did not receive hands-on training and 52\% performed $\leq 200$ procedures. Although most advanced fellowship programs include sufficient EUS volume for training purpose, this survey asserted some of the EUS training inadequacies and demonstrated areas of improvement.

In a systemic review evaluating the learning curve to achieve EUS competency, ${ }^{15}$ competency was achieved by 65-231 procedures for assessing T-staging accuracy for GI tumors and $30-40$ procedures for assessing EUS-FNA. The assessment of comprehensive EUS competency revealed that not all trainees achieved it; in fact, only four of 17 trainees achieved competency for 225-295 EUS procedures. These results demonstrated that the number of procedures required to acquire competency might need to be increased above ASGE recommendations.

Unfortunately, there have been few published reports regarding EUS training or the number of procedures required to achieve competence. ${ }^{16}$ Revision of the ASGE guidelines for EUS training might be needed since the above-mentioned survey and systemic review reported that performing 150 EUS procedures might be inadequate to help a fellow achieve competence and that at least 225 hands-on cases might need to be performed prior to the assessment of competency. ${ }^{17}$

\section{PROGRESS OF EUS TRAINING IN ASIAN COUNTRIES}

Contrary to the history of Western EUS training programs, 
formal EUS fellowship programs in Asian regions did not exist in the 2000s, except in Japan, Korea, and Taiwan. Most endoscopists rely on limited advanced endoscopy training programs at centers in Western countries to acquire EUS skills, and only a small number of endoscopists can benefit from privileged overseas training.

In the survey of EUS practices distributed to 87 endosonographers in the Asia-Pacific regions outside of Japan between August and October 2004, ${ }^{18}$ only $22.5 \%$ of respondents were formally trained through an overseas fellowship lasting $\geq 6$ months. Almost half of the EUS practitioners (49.3\%) were self-taught, more than half (59.2\%) were also trained through observing EUS procedures, and 25 (35.2\%) were taught in their own institution by experienced endosonographers. Another $12(16.9 \%)$ and five (7.0\%) received the same mode of training, but with a shorter duration of 3-6 months and $<3$ months, respectively. Most EUS practitioners (90\%) recognized that a formal EUS training fellowship with a minimum of 100 supervised procedures completed over a period of $\geq 6$ months was required to achieve acceptable EUS competence. This survey found that the major factors hindering the propagation of EUS in Asian countries are the sheer learning curve and insufficient training opportunities. To expand EUS utilization in Asia, the authors recommended a combination of advanced EUS training in centers of excellence and hands-on training using well-structured courses. To achieve the vision of encouraging EUS training in Asia, the Asian EUS Group (AEG) was organized in 2002 as an interest group that is not affiliated with any specialized society. AEG has offered a series of structured workshops. Between October 2012 and October 2013, 11 workshops in eight Asian countries were offered by a faculty of 20 regional EUS experts. ${ }^{19}$ Among the 11 workshops, the EUS training participants of six workshops evaluated the effectiveness of structured workshops in improving the knowledge and skills of EUS for the trainees by using written and hands-on tests with the Ikuma model. In the analysis of written tests before and after training from a total of 62 trainees, the overall mean scores improved significantly after versus before the training $(77.5 \% \pm 0.2 \%$ vs. $66.0 \% \pm 0.3 \%$, $p<0.0001)$. Among the 31 trainees who were randomly selected to undergo a skill test before and after training, there was a statistically significant advancement in the proportion of their successful localization of structures after hands-on training. In this study, structured training courses appeared to be an effective method of improving EUS knowledge and skills of aspiring endosonographers in the Asian region.

Asian countries have several advantages over Western countries for EUS training. Although Asian countries lack high-volume EUS centers compared to Western countries, one advantage is perhaps easier access to hands-on training on patients. After more than a decade of AEG effort and EUS procedural progress in Asian countries, more EUS local learning opportunities are available to aspiring endosonographers, albeit not entirely through formal fellowships, the supply of which remains short of demand. To overcome the shortage of training opportunities, a more concerted approach is now in place to fast-track the dissemination of EUS skills in Asia through well-structured short-term training programs. These programs are generally more intensive, are of shorter duration, and lack preceptorship.

\section{INFORMAL TRAINING PROGRAMS}

Several institutions across Western and Asian countries have offered systematic training courses in EUS. However, these programs provide limited exposure and certainly do not adequately train novices as independent endosonographers; additionally, the EUS learning curve is relatively lengthy. In addition to traditional fixed-term training and preceptorships, regular short-term intensive EUS training programs that provide training at various levels may help EUS practitioners improve and maintain their knowledges and skills. Ideally, although supervised hands-on training enables trainees to achieve competence more quickly than self-learning, mastering EUS, especially interventional EUS, is associated with an increased risk of complications and ethical issues. Therefore, informal EUS training and education programs for achieving EUS training competence are required to improve training and reduce the number of supervised examinations.

There are many ways to learn EUS other than supervised practice on actual patients. Theoretical knowledge can be acquired from lectures, textbooks, atlases, slides, videotapes, digital video discs, interactive compact discs, and websites. Informal EUS training is generally based on 1- or 2-day intensive workshops, including didactic lectures, skills demonstrations by expert practitioners through live video-streaming of procedures, and hands-on learning using animal or phantom models. ${ }^{4}$ Most academic and tertiary referral centers have offered both formal and informal EUS training models for over a decade. However, these informal learning methods simply represent useful adjuncts to formal training and should not be used as a substitute for a more formal supervised training experience.

\section{NONHUMAN TRAINING MODELS}

Available nonhuman models to help facilitate EUS training include computer-based simulators, phantoms, ex vivo mod- 
els, and live animal models. ${ }^{20}$

Among computer-based endoscopic simulators for GI endoscopy, GI-Mentor using an EUS module (Simbionix Ltd., Tel Hashomer, Israel) and EUS Meets Voxel-Man (Voxel-Man Group, Hamburg, Germany) are used for training in EUS. ${ }^{21,22}$ EUS Mentor is a computer-based endoscopic simulator platform that allows trainees to gain experience by manipulating the scope and wheels and provides realistic radial and linear-array EUS images based on human anatomy. EUS Meets Voxel-Man is an interactive anatomic simulation program that provides three-dimensional anatomy on linear-array EUS images. ${ }^{20,23}$ More advanced models suitable for teaching interventional EUS have recently been made available. A demonstration of the various steps for typical EUS-guided biliary drainage is now achievable using the Mumbai EUS 3D model (Prototype, Mumbai, India), a stereolithography, 3D printing bile duct prototype. ${ }^{24}$ Dhir et al. showed a $100 \%$ success rate of needle puncture and tract dilation, $82.4 \%$ for wire manipulation, and $80 \%$ for stent placement. ${ }^{24} \mathrm{~A}$ major limitation of the current simulated models is the lack of haptics.

EUS and EUS-FNA phantoms (Olympus, Tokyo, Japan) consist of a longitudinal body with a hole in the center and different silicon block of types and sizes that simulate the esophagus and lymph nodes or cystic lesions, respectively. This model is simple and easy to use and transport. Various models are available to represent subepithelial tumors, various cancer invasion depths, radial and linear EUS models of the pancreatobiliary system, and EUS-FNA. Although these phantoms do not adequately simulate actual human anatomy, they could help trainees learn the basic EUS and EUS-FNA techniques, especially manipulating and positioning the echoendoscope and FNA needle.

The Ikuma model was designed through collaboration between Olympus Medical Systems Corporation (Tokyo, Japan) and Kyoto Kagaku (Kyoto, Japan) to simulate real-time human anatomy. Trainees can practice navigational skills with this model, but it cannot simulate the tactile feeling of working with real patients. An ex vivo animal model such as the EUS RK model offers enhanced realistic feeling and EUS-FNA is feasible using normal EUS equipment. However, this model does not simulate blood flow.

After hands-on tutelage by an expert mentor, practicing EUS on live animals is probably the next best way to acquire practical EUS skills. Live animal models are the most realistic simulators and offer the best training experience. The swine model is the most common animal model for EUS learning. Swine models resemble human anatomy and human-like EUS images can be obtained from transgastric scanning. EUS training using live animal models significantly improves trainee performance, confidence, and procedural comfort with returning to real patient examinations. However, animal models are expensive and difficult to set up, and using them means sacrificing animals.

Although each model has its own advantages and limitations, its value is complementary rather than competitive. Unfortunately, these simulators are not readily available at most institutions due to high cost and regional needs. Clinical trials evaluating the efficacy of simulators in EUS training are lacking. Further studies of the efficacy of various EUS training models on technical and clinical outcomes are still needed.

\section{CONCLUSIONS}

EUS has become an important imaging tool for the evaluation of a variety of GI disorders. It is a challenging endoscopic procedure that requires both cognitive and technical skills beyond the general scope of standard endoscopic procedures. New EUS procedures and clinical applications are constantly advancing. Obtaining competent EUS training may be challenging since EUS is highly operator-dependent and training on actual patients can be associated with an increased risk of complications and ethical issues. The British guidance on EUS services in the UK specifically states, 'There is no role for the self-teaching of EUS through trial and error'. Even after structured training, an acceptable diagnostic accuracy rate can be achieved only after the execution of a certain number of examinations. As the demand for expert endosonographers continues to increase, training guidelines must be critically revised to ensure the availability of well-trained and competent future endosonographers.

\section{Conflicts of Interest}

The author has no financial conflicts of interest.

\section{REFERENCES}

1. Godfrey EM, Rushbrook SM, Carroll NR. Endoscopic ultrasound: a review of current diagnostic and therapeutic applications. Postgrad Med J 2010;86:346-353.

2. Shami VM, Waxman I. Technology insight: current status of endoscopic ultrasonography. Nat Clin Pract Gastroenterol Hepatol 2005;2:38-45.

3. Parada KS, Peng R, Erickson RA, et al. A resource utilization projection study of EUS. Gastrointest Endosc 2002;55:328-334.

4. Wong JYY, Kongkam P, Ho KY. Training in endoscopic ultrasonography: an Asian perspective. Dig Endosc 2017;29:512-516.

5. Van Dam J, Brady PG, Freeman M, et al. Guidelines for training in electronic ultrasound: guidelines for clinical application. From the ASGE. American Society for Gastrointestinal Endoscopy. Gastrointest Endosc 1999;49:829-833.

6. Caletti G, Togliani T, Fusaroli P. EUS education in EU. Dig Endosc 2004;16(Suppl 2):S142-S143.

7. Chang KJ. Endoscopic ultrasound education in the USA. Dig Endosc 2004;16(Suppl 2):S138-S141. 
8. Meenan J, Anderson S, Tsang S, Reffitt D, Prasad P, Doig L. Training in radial EUS: what is the best approach and is there a role for the nurse endoscopist? Endoscopy 2003;35:1020-1023.

9. Lightdale CJ. EUS training in the USA. Endoscopy 1998;30(Suppl 1):A19-A21.

10. Rösch T. State of the art lecture: endoscopic ultrasonography: training and competence. Endoscopy 2006;38(Suppl 1):S69-S72.

11. Eisen GM, Dominitz JA, Faigel DO, et al. Guidelines for credentialing and granting privileges for endoscopic ultrasound. Gastrointest Endosc 2001;54:811-814.

12. Meenan J, Harris K, Oppong K, et al. Service provision and training for endoscopic ultrasound in the UK. Frontline Gastroenterol 2011;2:188 194.

13. Arya N, Sahai AV, Paquin SC. Credentialing for endoscopic ultrasound a proposal for Canadian guidelines. Endosc Ultrasound 2016;5:4-7.

14. Azad JS, Verma D, Kapadia AS, Adler DG. Can U.S. GI fellowship programs meet American Society for Gastrointestinal Endoscopy recommendations for training in EUS? A survey of U.S. GI fellowship program directors. Gastrointest Endosc 2006;64:235-241.e2.

15. Shahidi N, Ou G, Lam E, Enns R, Telford J. When trainees reach competency in performing endoscopic ultrasound: a systematic review. Endosc Int Open 2017;5:E239-E243.

16. Wani S, Coté GA, Keswani R, et al. Learning curves for EUS by using cumulative sum analysis: implications for American Society for Gas- trointestinal Endoscopy recommendations for training. Gastrointest Endosc 2013;77:558-565.

17. ASGE Standards of Practice Committee, Faulx AL, Lightdale JR, et al Guidelines for privileging, credentialing, and proctoring to perform GI endoscopy. Gastrointest Endosc 2017;85:273-281

18. Ho KY. Survey of endoscopic ultrasonographic practice and training in the Asia-Pacific region. J Gastroenterol Hepatol 2006;21:1231-1235.

19. Wang MH, Dy F, Vu VK, et al. Structured endoscopic ultrasonography (EUS) training program improved knowledge and skills of trainees: results from the Asian EUS Group. Dig Endosc 2015;27:687-691.

20. Kim GH, Bang SJ, Hwang JH. Learning models for endoscopic ultrasonography in gastrointestinal endoscopy. World J Gastroenterol 2015;21:5176-5182.

21. Bar-Meir S. A new endoscopic simulator. Endoscopy 2000;32:898-900.

22. Matsuda K, Tajiri H, Hawes RH. How shall we experience EUS and EUS-FNA before the first procedure?: the development of learning tools. Dig Endosc 2004;16(Suppl 2):S236-S239.

23. Burmester E, Leineweber T, Hacker S, Tiede U, Hütteroth TH, Höhne KH. EUS meets Voxel-Man: three-dimensional anatomic animation of linear-array endoscopic ultrasound images. Endoscopy 2004;36:726-730.

24. Dhir V, Itoi T, Fockens P, et al. Novel ex vivo model for hands-on teaching of and training in EUS-guided biliary drainage: creation of "Mumbai EUS" stereolithography/3D printing bile duct prototype (with videos). Gastrointest Endosc 2015;81:440-446. 